\title{
ON A GENERALIZATION OF THE ABSTRACT MORSE COMPLEX AND ITS APPLICATIONS
}

\author{
SUK HO HONG
}

\section{Introduction}

Klingenberg refers in [4] the fact that the homology group of the space $\Lambda$ of closed $H^{1}$ curves on a manifold is isomorphic to that of the Morse complex. In this paper, we generalize the fact above and at the same time give a proof to it through cell decomposition method under a strong non degeneracy condition.

We first introduce so-called generalized Morse complex on a space $X$ with an action of Lie group $G$ and an invariant energy function $E$ on $X$. The case of the space $\Lambda$ of closed curves is obviously obtained through $G=S^{1}$.

Next we apply the Morse complex argument to the space $\Lambda$, where the isotropy group is closely related to the multiplicity. And we find the cycle $Z(c)$ constructed by Shikata-Klingenberg [1] is at most finite order in the homology of the Morse complex. Thus from a close investigation of the order of the cycle $Z(c)$ on $H_{*}(X)$, we deduce a relation between the torsion and the divisibility of multiplicities of a certain geodesic.

We would like to thank Professor Y. Shikata for many valuable discussions who gives me many helpful insight into the field of Morse complex.

\section{§1. On $G$-action which generalizes $S^{1}$-action on $\Lambda$}

1-1. Let $X$ be a $C^{r+1}$-manifold $(r \geq 0)$ with a $G$-action of a compact Lie group such that the isotropy group $I(p)$ at $p \in X$ is discrete for any $p \in X$. Suppose $X$ admits an invariant Morse function $E$, i.e.,

$$
E: X \longrightarrow R
$$

is $c^{r}$-function such that $E(g p)=E(p)$ for any $g \in G$ and let $\varphi$ be the gradient flow of $E$, then $\varphi$ is $G$-equivariant:

Received August 19, 1983. 


$$
\varphi(g p)=g \varphi(p)
$$

for any $g \in G$ and $p \in X$.

For a critical point $c$ of $E$, we set

$$
\begin{aligned}
& S(c)=\left\{p \in X: \varphi_{s}(p) \longrightarrow c \text { as } s \longrightarrow \infty\right\} \\
& U(c)=\left\{p \in X: \varphi_{s}(p) \longrightarrow c \text { as } s \longrightarrow-\infty\right\}
\end{aligned}
$$

then $S(c)$ and $U(c)$ are called the stable and unstable manifolds respectively.

Theorem 1. If $c$ is non-degenerated, $\operatorname{Codim} G \cdot S(c)=\operatorname{index} c$.

Proof. Denote by $T_{p}(M)$ the tangent space of a submanifold $M$ at $p$, then from the non degeneracy assumption above we have a natural spliting

$$
T_{p}(U(c)) \oplus T_{p}(G \cdot S(c))=T_{p}(X)
$$

since

$$
\operatorname{dim} U(c)=\operatorname{index} c
$$

we have

$$
\operatorname{codim} G \cdot S(c)=\operatorname{index} c .
$$

We choose from each $G$-orbit $G \cdot c$ of a critical point $c$, a representative $c$ and call them a pure critical point representing $c$ and denote the set of pure critical point by $\Gamma$.

We introduce a polar coordinate system $(u, t)_{\mathrm{c}}$ for $u \in S\left(T_{c}(U(c))\right.$ and $t \in(0, \infty)$ where $S\left(T_{c}(U(c))\right),=\left\{u \in T_{c}(U(c))\right.$, $\left.\|u\|=1\right\}$ in the unstable manifold $U(c)$ of a critical point $c$ by mapping $(u, t)_{c}$ onto $\varphi_{t}(u)$. We deduce the following property for the polar coordinate easily:

LEMMA 2. $g(u, t)_{c}=(g u, t)_{g c}$ where we used the notation gu also for the G-action on the tangent space.

It is obvious that if any two flows $\varphi_{t}(u), \varphi_{t^{\prime}}\left(u^{\prime}\right)\left(u \in T_{c}(U(c)), u^{\prime} \in T_{c^{\prime}}(U(c))\right)$ have an intersection for finite $t$, $t^{\prime}$, then they are agree entirely, therefore we may refer this fact as follows:

LEMmA 3. If $(u, t)_{c}=\left(u^{\prime}, t^{\prime}\right)_{c^{\prime}}$ for $c^{\prime} \in G \cdot c$, then

$$
u=u^{\prime}, t=t^{\prime} \quad \text { and } \quad c=c^{\prime} \text {. }
$$

We refer the following property $(\mathrm{P})$ at the strong non degeneracy of $E$ : 
(P) : All the critical point $c$ are non degenerate and for any critical points $c, c^{\prime}$, the stable and unstable manifolds have a generic intersection.

1-2. We compute $H_{*}(X)$ through a cell decomposition of $X$. We first decompose $G \cdot U(c)$ into cells: Consider the covering space

$$
\pi: G \longrightarrow G / I(c)
$$

with the right hand $I(c)$-action and decompose the base manifold $G / I(c)$ into cells $\{\bar{\Delta}\}$ such that the covering $\pi$ is trivial over each simplex $\bar{\Delta} \in\{\bar{\Delta}\}$. Then $A(\bar{\Delta})=\pi^{-1}(\bar{\Delta})$ splits into a disjoint union $\left\{A_{i}(\bar{\Delta})\right\}$ of homeomorphic cells in $G$ on which $I(c)$ acts effectively and transitively from the right. We choose and fix a representative $A_{c}(\bar{\Delta})$ from the inverse image $\left\{A_{i}(\bar{\Delta})\right\}$ of each cell $\bar{\Delta}$ in $\{\bar{d}\}$.

Lemma 4. If there exist points $p, p^{\prime}, q, q^{\prime}$ such that

$$
p \in A_{c}(\bar{\Delta}), \quad p^{\prime} \in A_{c}\left(\bar{\Delta}^{\prime}\right), \quad q, q^{\prime} \in U(c)
$$

and

$$
p q=p^{\prime} q^{\prime} \quad \text { for cells } \bar{\Delta}, \bar{\Delta}^{\prime} \in\{\bar{\Delta}\}
$$

then we have

$$
\bar{\Delta}=\bar{\Delta}^{\prime}, p=p^{\prime} \text { and } q=q^{\prime} .
$$

In fact, in the polar coordinate on $U(c)$, we have

$$
p(u, t)_{c}=p^{\prime}\left(u^{\prime}, t^{\prime}\right)_{c}
$$

therefore from Lemma 3, we see

$$
p c=p^{\prime} c
$$

that is

$$
p=p^{\prime} x, \quad x \in I(c) .
$$

Since $\pi$ is $I(c)$-covering, we have $x=\mathrm{id}$.

Proposition 5. The cell $A_{c}(\bar{\Delta})$ in $G$ defines a cell $A_{c}(\bar{\Delta}) \cdot U(c)$ in $G \cdot U(c)$ which is homeomorphic to $A_{c}(\bar{A}) \times U(c)$ in the interior.

Proof. If $(p, q),\left(p^{\prime}, q^{\prime}\right) \in A_{c}(\bar{\Delta}) \times U(c)$ are mapped onto the same point through the multiplication, we have immediately from Lemma 4 that

$$
p=p^{\prime} \text { and } q=q^{\prime} .
$$


Proposimion 6. The cells $A_{c}(\bar{\Delta}) \cdot U(c), A_{c}\left(\bar{\Delta}^{\prime}\right) \cdot U(c)$ have no interior intersection for $\bar{\Delta} \neq \bar{\Delta}^{\prime}$.

Proof. It is also obvious from Lemma 4 that the existence of the interior intersection

$$
p q=p^{\prime} q^{\prime} \quad \text { for } \quad p \in A_{c}(\bar{\Delta}), p^{\prime} \in A_{c}\left(\bar{\Delta}^{\prime}\right),
$$

$q, q^{\prime} \in U(c)$ implies $p=p^{\prime}, \bar{\Delta}=\bar{\Delta}^{\prime}$.

Since $U(c)=g U(c)$ for any $g \in I(c)$ as sets we finally see that

$$
\begin{aligned}
G \cdot U(c) & =\bigcup_{\{\bar{A}\}} \bigcup_{i} A_{i}(\bar{\Delta}) \cdot U(c) \\
& =\bigcup_{\{\bar{d}\}} \bigcup_{g \in I(c)} A_{c}(\bar{\Delta}) \cdot g U(c) \\
& =\bigcup_{\{\bar{A}\}} A_{c}(\bar{\Delta}) \cdot U(c)
\end{aligned}
$$

that is, the cells $A_{c}(\bar{\Delta}) \cdot U(c)$ for $\bar{\Delta} \in\{\bar{\Delta}\}$ cover $G \cdot U(c)$.

THEOREM 7. The cells $A_{c}(\bar{\Delta}) \cdot U(c)$ give a cell decomposition of $G \cdot U(c)$.

We see that a subdivision of the decomposition of $G \cdot U(c)$ induces a decomposition on $b d(G \cdot U(c))$ as follows: First, property (P) yields that $S\left(T_{c}(U(c))\right)$ is divided into cells by its intersection with the (weak) stable manifold $S\left(c_{-}\right)$of critical points $c_{-}$of lower indexes than $c$, in fact the intersection

$$
S\left(T_{c}(U(c))\right) \cap S\left(c_{-}\right)
$$

is an open submanifold $S\left(T_{c}(U(c))\right)$ of dimension

$$
\text { index } c-\operatorname{index} c_{-}-1
$$

and the boundary of each one of the submanifold again splits into a union of submanifolds of this kind.

Thus taking product by small cell $\Delta \subset G$ to these cells, we can divide $\Delta \cdot S\left(T_{c}(U(c))\right)$ into cells. Therefore for a sufficiently fine decomposition $\{\bar{\Delta}\}$ of $G$ we see that the decomposition of $A_{c}(\bar{\Delta}) \cdot S\left(T_{c}(U(c))\right)$ defines a natural decomposition of $A_{c}(\bar{\Delta}) \cdot U(c)$ through the polar coordinate. Take a decomposition $\{\bar{\Delta}\}$ of $G$ so fine that the covering projection $\pi: G \rightarrow G / I\left(c_{-}\right)$ is trivial over $\bar{\Delta}$ for any pure critical point $c_{-}$such that $S\left(c_{-}\right) \cap S\left(T_{c}(U(c))\right)$ $\neq \phi$, then we see that $\{\bar{\Delta} \cdot b d U(c)\}$ decomposes $G \cdot b d U(c)$ into cells, because $b d U(c)$ is $\omega$-limit of $S\left(T_{c}(U(c))\right)$. 
Let $X(n)$ denote the union of (weak) unstable manifolds over pure critical points of index lower than $n$ or equal to $n$.

$$
\begin{aligned}
& X(n)=\bigcup_{c \in \Gamma(n)} G \cdot U(c) \\
& \Gamma(n)=\{c \in \Gamma, \quad \text { index } c \leq n\} .
\end{aligned}
$$

Then it is easy to see that $X(n)$ can be decomposed into cells in the method above and

$$
X=\bigcup_{n} X(n), \quad X(n) \subset X(n+1) .
$$

Since any $k$-submanifold in $X$ is pushed down into $X(k)$ by the flow.

THEOREM 8. The homology $H_{k}(X)$ may be computed as the homology $H_{k}(X(n))$ of $X(n)(k<n)$ which is obtained as homology of a cell decomposition given by a subdivision of the cells $A_{c}(\bar{\Delta}) \cdot U(c)$.

1-3. We construct an abstract chain complex $\mathscr{M}$ which is equivalent to the chain group over the cell complex above and we call it a generalized Morse complex. We fix an orientation on each cell of $\{\bar{\Delta} \cdot U(c)\}$ by choosing an orientation in $U(c)$ and also one in $\bar{\Delta} \in\{\bar{\Delta}\}_{c}$ for each pure critical point $c \in \Gamma$. We then have an graded chain group $C(X)$ of oriented cell $\{\bar{\Delta} \cdot U(c)\}$ by defining

$$
\begin{aligned}
\operatorname{deg} \bar{\Delta} \cdot U(c) & =\operatorname{dim} \bar{\Delta} \cdot U(c) \\
& =\operatorname{dim} \bar{\Delta}+\operatorname{index} c .
\end{aligned}
$$

Let $X^{n}$ be the union of cells in $X(m)$ of dimension lower than or equal to $n(n \geq m)$ and take the boundary operator $\partial$ in the exact sequence for the triple $\left(X^{n}, X^{n-1}, X^{n-2}\right)$ :

$$
\text { ว: } H_{n}\left(X^{n}, X^{n-1}\right) \longrightarrow H_{n-1}\left(X^{n-1}, X^{n-2}\right)
$$

then it is known that $C_{n}(X)=H_{n}\left(X^{n}, X^{n-1}\right)$ and $C(X)$ turns out to be a chain complex together with the boundary $\partial$ (see [2], [6]), whose homology is equal to that of $X(m)$, thus we have

Proposition 9. Under the non degeneracy condition, we have a chain complex $C(X)$ over graded cells $\{\bar{\Delta} \cdot U(c)\}$ so that

$$
H_{*}(C(X))=H_{*}(X) \text {. }
$$

CoROllary 10. Under the same non degeneracy condition above, we 
see that the homology $H_{*}(C(X))$ of the cell complex is independent of the cell decomposition of $X$, especially that of $G$.

In order to describe the boundary operator $\partial$, we start with a small cell $e=\tilde{\Delta} \cdot U(c)$ in $H_{n}\left(X^{n}, X^{n-1}\right)$, which is represented as the image of a (relative) product homeomorphism $\varphi=\varphi_{1} \times \varphi_{2}$ of

$$
\varphi_{1}: I^{k} \longrightarrow X
$$

and

$$
\varphi_{2}: I^{m} \longrightarrow X
$$

such that $\pi: G \rightarrow G / I(c)$ is trivial over $\varphi_{1}(I)=\tilde{\Delta}$ :

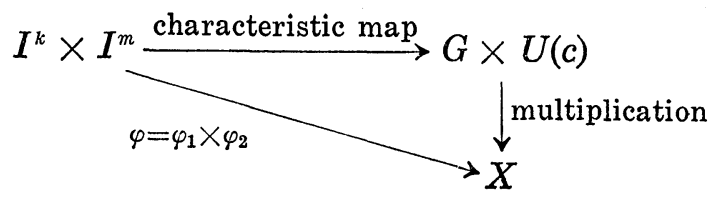

Since $\varphi_{*}$ commutes with the boundary homeomorphism, we see that $\partial e=$ $j_{*} \varphi_{*} \partial_{*} f$ for the fundamental class $f$ in $H_{n}\left(I^{k} \times I^{m}\right.$, bd $\left.\left(I^{k} \times I^{m}\right)\right)$, as is seen from the following diagram:

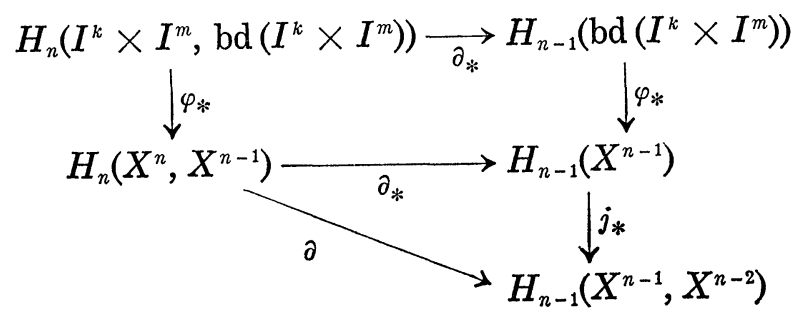

The fundamental class $f$ splits into a cross product $f_{1} \times f_{2}$ of

$$
\begin{aligned}
& f_{1} \in H_{k}\left(I^{k}, \operatorname{bd} I^{k}\right), \\
& f_{2} \in H_{m}\left(I^{m}, \operatorname{bd} I^{m}\right) \\
& k=n-m=\operatorname{dim} \tilde{\Delta} \\
& m=\operatorname{index} c
\end{aligned}
$$

corresponding to $\tilde{\Delta}$ and to $U(c)$, respectively, therefore from the naturality as the boundary formula of the cross product, we have that

$$
\begin{aligned}
\partial e & =j_{*} \varphi_{*} \partial f \\
& =j_{*} \varphi_{*}\left(\partial_{*} f_{1} \times f_{2}+(-1)^{k} f_{1} \times \partial_{*} f_{2}\right)
\end{aligned}
$$




$$
\begin{aligned}
& =\left(j_{*} \varphi_{1^{*}} \partial_{*} f_{1} \times j_{*} \varphi_{2^{*}} f_{2}\right)+(-1)^{k}\left(j_{*} \varphi_{1^{*}} f_{1}\right) \times\left(j_{*} \varphi_{2^{*}} \partial \partial_{*} f_{2}\right) \\
& =\partial e_{1} \times e_{2}+(-1)^{k} e_{1} \times \partial e_{2}
\end{aligned}
$$

Here the classes

$$
\begin{aligned}
& e_{1}=j_{*} \varphi_{1^{*}} f_{1} \in H_{k}\left(X^{k}, X^{k-1}\right) \\
& e_{2}=j_{*} \varphi_{2^{*}} f_{2} \in H_{m}\left(X^{m}, X^{m-1}\right)
\end{aligned}
$$

may be regarded as the classes representing $\tilde{\Delta}$ and $U(c)$ respectively. Moreover we may replace the cross product above by the multiplication of $G$ on $X$ because every cell under consideration acts effectively on $U(c)$, thus we see that

Proposition 11. The boundary operator $\partial$ in the cell decomposition of Theorem 8 in Section 1 satisfies that

$$
\partial(\tilde{\Delta} \cdot U(c))=(\partial \tilde{\Delta}) \cdot U(c)+(-1)^{k} \tilde{\Delta} \cdot \partial U(c), \quad \text { where } k=\operatorname{dim} \tilde{\Delta} .
$$

Finally we investigate $\tilde{\Delta}, U(c)$ geometrically.

They may be considered as the homology classes represented as the classes of the boundaries

$$
\begin{aligned}
& \partial \tilde{\Delta}=\partial e_{1}=j_{*}\left(\varphi_{1}\right)_{*} \partial_{*} e_{1} \in H_{k-1}\left(X^{k-1}, X^{k-2}\right) \\
& \partial U(c)=\partial e_{2}=j_{*}\left(\varphi_{2}\right)_{*} \partial_{*} U(c) \in H_{m-1}\left(X^{m-1}, X^{m-2}\right) .
\end{aligned}
$$

Therefore $\partial U(c)$ can be regarded as the sum of $(m-1)$ cells appearing on the boundary of $U(c)$ with the suitable coefficient, which we can count as the intersection number of $S\left(T_{c}(U(c))\right.$ ) with the (weak) stable manifold $S\left(\tilde{\Delta} c_{-}\right)$of codimension $m-1$ for a cell $\tilde{\Delta} \in G$ of dimension index $c_{-}$ $(m-1)$.

LEMma 12. Let $\left[\tilde{\Delta} c_{-}, c\right]$ be the intersection number of $\left.S\left(T_{c}(U(c))\right)\right]$ and the stable manifold $S\left(\tilde{\Delta} c_{-}\right)$of codimension $m-1$, then we have

$$
\partial U(c)=\sum\left[\tilde{\Delta} c_{-}, c\right] \tilde{\Delta} U\left(c_{-}\right) .
$$

We introduce an abstract chain complex $\mathscr{M}$ over the set $\Gamma$ of the"pure critical points as the chain group generated over formal elements

$$
\{\tilde{\Delta} c / \tilde{\Delta}: \text { cell in } G, c \in \Gamma\}
$$

with the degree given by

$$
\text { degree } \tilde{\Delta} c=\operatorname{dim} \tilde{\Delta}+\operatorname{index} c
$$

and define the boundary operator $\partial$ as follows: 


$$
\begin{aligned}
& \partial c=\sum\left[\tilde{\Delta} c_{-}, c\right] \tilde{\Delta} c_{-} \\
& \partial \tilde{\Delta} c=\partial \tilde{\Delta} c+(-1)^{k} \tilde{\Delta} \partial c \quad \text { where } k=\operatorname{dim} \tilde{\Delta} .
\end{aligned}
$$

Since we see easily that the chain complex $\mathscr{M}$ is chain homotopic to $C(X)$, we deduce the following from Lemma 12, Propositions 9, 11.

Theorem 13. $H_{*}(\mathscr{M})=H_{*}(X)$.

\section{§2. Relations to torsion and divisibility}

2-1. In case of the space $\Lambda$ of closed curve, we have a natural $S^{1}$ action on $\Lambda$ through the action on the parameter;

$$
\theta \cdot \alpha(t)=\alpha(\theta+t) \quad t, \theta \in S^{1}, \quad \alpha \in \Lambda .
$$

If we remove the point curves $\Lambda_{0}$ from $\Lambda$, we have the $S^{1}$-action on $\Lambda-\Lambda_{0}$ such that the isotropy $I(x)$ is discrete for any $x \in \Lambda-\Lambda_{0}$ thus we may apply our method to the case $X=\Lambda-\Lambda_{0}, G=S^{1}$.

In this case, we have a well known relation between the order of Iso $(x)$ and the multipilicity $m(x)$ of $x$ defined as the maximal number $m$ so that

$$
x=\alpha \cdots \alpha=\alpha^{m} \quad \text { for some } \alpha \in \Lambda .
$$

LEMMA 14. ord $I(x)=m(x)$.

We notice that when we consider the $S^{1}$-action on the Morse complex $\mathscr{M}$, then also have a notion of isotropy Iso $(x)$ for a chain $x \in C$. In particular for a chain represented by a critical ponit $c$, we have Iso $(c)$ other than $I(c)$.

LEMma 15. ord Iso $(c)=\operatorname{ord} I(c)$ or 2ord $I(c)$

$$
=m(c) \text { or } 2 m(c) \text {. }
$$

In fact, if the multiplication by $g \in I(c)$ on $U(c)$ preserves the orientation in $U(c)$, we have the first case, otherwise we take double in order to preserve the orientation and we have the second case.

On the other hand, Klingenberg constructed a energy function $E$ on the space $A$ which satisfies the condition (C). (cf. Klingenberg [4]). Therefore if we assume further the strong degeneracy on $E$, we may apply Theorem 13 to the space $\Lambda-S\left(\Lambda_{0}\right)$, where $S\left(\Lambda_{0}\right)$ denotes the stable manifold over $\Lambda_{0}$ and we reproduce the Klingenberg's anouncement [4] on the homology of $\Lambda-S\left(\Lambda_{0}\right)$ with $S^{1}$-action. 
THEOREM 16. The homology $H_{*}\left(\Lambda-S\left(\Lambda_{0}\right)\right)$ of $\Lambda-S\left(\Lambda_{0}\right)$ is obtained as the homology of the Morse complex associated with $\Lambda-S\left(\Lambda_{0}\right)$ and $E$, provided that $E$ satisfies the strong non degeneracy condition.

It may be possible to weaken the strong non degeneracy condition to a weak non degeneracy, that is, only assuming the non degeneracy of each critical point, for this we return in near future.

Our purpose in the remaining is to investigate a relation between a torsion property of homology $H_{*}(X)$, (reduced to the Morse complex) and a behavior of the multiplicities which is related to the order of isotropy as an application of what we have discussed.

Our point is that we can deduce a type of divisibility even for the Finsler case provided the strong non degeneracy because our method is entirely topological and does not use the $\vartheta$-action which comes from Riemannian structure.

2-2. We investigate a torsion property of a cycle $Z(c)$ in $\mathscr{M}$ constructed by Shikata-Klingenberg [1]. We quickly review here how $Z(c)$ is constructed over a pure critical point $c \in \Gamma$. Let $\bar{m}$ be the order of isotropy of $c$, then we have

$$
1 / \bar{m} \cdot c=c,
$$

hence

$$
1 / \bar{m} \cdot \partial c=\partial c .
$$

Thus we have an invariant chain $\partial c$ in $\mathscr{M}$ under the action of a subgroup $G(\bar{m})$ of $S^{1}$ generated by $1 / \bar{m}$ and therefore we can split $\partial c$ into a sum of invariant chains $x_{i}$ which is invariant under the action of a subgroup $H_{i} \supset G(\bar{m})$ :

$$
\partial c=\sum_{1}^{n} x_{i}
$$

Then the fact that

$$
h_{i} x_{i}=x_{i} \quad \text { for } h_{i} \in H_{i}
$$

implies that

$$
\partial\left(\left(1-h_{1}\right) \cdots\left(1-h_{n}\right) c\right)=0,
$$

yielding a cycle

$$
Z(c)=\left(1-h_{1}\right) \cdots\left(1-h_{n}\right) c
$$


In order to investigate a further property of the cycle $Z(c)$, we consider the case $n=1, H_{1} \supseteq G(\bar{m})$.

Lemma 17. Let $h=1$ /ord $H_{1}$ then

$$
Z(c)=(1-h) c
$$

is at most a torsion element of ord $\left(H_{1}\right)$.

In fact, take

$$
\Delta=[0, h]
$$

and let

$$
y=\Delta c
$$

then we have

$$
\begin{aligned}
\partial y & =\partial \Delta \cdot c-\Delta \partial c \\
& =(1-h) c-\Delta x_{1} \\
& =Z(c)-\Delta \cdot x_{1} .
\end{aligned}
$$

Since $x_{1}$ is $H_{1}$-invariant, it is expressed as a sum over $H_{1}$ :

$$
x_{1}=\sum_{k \in H_{1}} k u, \quad u \in \mathscr{M} .
$$

Therefore

$$
\Delta x_{1}=\sum_{k \in H_{1}} k \cdot \Delta u=\left(\sum_{k \in H_{1}} k \Delta\right) \cdot u
$$

may be expressed as $\Delta x_{1}=S^{1} \cdot u$.

Thus we have

$$
\partial y=Z(c)-S^{1} \cdot u \text {. }
$$

On the other hand, consider $v=S^{1} \cdot c$ then we see that

$$
\begin{aligned}
\partial v & =\partial S^{1} \cdot c-S^{1} \cdot \partial c \\
& =-S^{1}\left(\sum_{k \in H_{1}} k \cdot u\right) \\
& =-\sum_{k \in H_{1}} k\left(S^{1} \cdot u\right) \\
& =-\left(\sum_{k \in H_{1}} k\right) \cdot S^{1} \cdot u \\
& =-\left(\operatorname{ord} H_{1}\right) S^{1} \cdot u .
\end{aligned}
$$

Hence we have that

$$
\left(\operatorname{ord} H_{1}\right) \partial y=\left(\operatorname{ord} H_{1}\right) Z(c)+\partial v .
$$


indicating that $Z(c)$ is at most of ord $\left(H_{1}\right)$ torsion in $H_{*}(\mathscr{M})$.

Next we take the case $n=2$,

$$
H_{1} \supsetneq G(\bar{m}), \quad H_{2} \supsetneq G(\bar{m})
$$

and

$$
H_{1} \cap H_{2} \neq H_{1}, H_{2} .
$$

LEMMA 18. Let

$$
h_{1}=1 / \text { ord } H_{1}, \quad h_{2}=1 / \text { ord } H_{2}
$$

and $Z(c)$ is of the form

$$
Z(c)=\left(1-h_{1}\right)\left(1-h_{2}\right) c
$$

then it is zero in $H_{*}(\mathscr{M})$.

In fact, take $\Delta=[0, h]$ and let

$$
y=\Delta\left(1-h_{2}\right) c
$$

then we see that

$$
\begin{aligned}
\partial y & =Z(c)-\Delta\left(1-h_{2}\right) \partial c \\
& =Z(c)-\Delta\left(1-h_{2}\right)\left(x_{1}+x_{2}\right) \\
& =Z(c)-\Delta\left(1-h_{2}\right) x_{1} \\
& =Z(c)-\left(1-h_{2}\right) S^{1} \cdot u
\end{aligned}
$$

by the same $u$ and by the same reasoning as in the case 1 . Thus we see that

$$
\partial y=Z(c) .
$$

In general, from a similar computation, we see easily that for $n \geqq 2$, the homology class $Z(c)$ is zero, also we may remark that for the case $n=1$ the homology classes $(1-h) Z(c)$ is zero.

In [1] Shikata-Klingenberg deduced a modified divisibility lemma using a chain bounding the cycle $Z(c)+\vartheta Z(c)$, for the involution $\vartheta$ in $\Lambda$ keeping $E$ invariant. Thus their theory is related to the Riemannian structure of the underlying manifold at this point. But we can cut this point off from the Riemannian structure by taking $Z(c)$ or $(1-h) Z(c)$.

Proposition 19. We may apply Shikata-Klingenberg theory to the cycle $Z(c)$ or $(1-h) Z(c)$ to have the divisibility lemma in the modified form 
even in case we do not have the involution $\vartheta$, like in non symmetric Finsler space.

Remark 1. Shikata-Klingenberg theory uses $\pi_{1}(\Lambda)=0$ on the way, therefore Katok's Finsler example on $S^{2}$ has nothing to do with the proposition above.

Remark 2. Shikata-Klingenberg's modified divisibility lemma is roughly as follows: Under a certain non degeneracy assumption as $\pi_{1}(\Lambda)$ $=0$, there exists a series $\left\{c_{i}\right\}$ of critical points in $\Lambda$, so that

$$
m\left(c_{i}\right) \mid 2 m\left(c_{i+1}\right) \text { or } m\left(c_{i+1}\right) \mid 2 m\left(c_{i}\right)
$$

where the $m(c)$ is the multiplicity of the curve $c$ in $A$ and is related to the order of isotropy $I(c)$.

\section{REFERENCES}

[1] W. Klingenberg and Y. Shikata, On a proof of the divisibility lemma, Closed geodesics in Tokyo University Symposium 1982.

[2] - On the existence theorems of infinitely many closed geodesics, Proc. Int. Conference on Topology, Moscow 1979.

[ 3 ] W. Klingenberg, Riemannian geometry, Walter de Gruyter 1982.

[ 4 ] — Lectures on closed geodesics, Springer Verlag 1978.

[ 5 ] Glen E. Bredon, Equivariant Cohomology Theories, Springer Verlag 1967.

[6] E. Spanier, Algebraic topology, McGraw Hill 1966.

[7] J. Milnor, Lectures on the $h$-cobordism theorem, Princeton University Press 1965.

[ 8 ] —, Morse theory, Princeton University Press 1963.

[ 9 ] R. Thom, Quelques propriétés globales des variétés differentiables Comment. Math. Helv., 281954.

Department of Mathematics

Nagoya University

Nagoya 464 Japan

Current address:

Department of Mathematics

Kon-kuk University

Seoul, Korea 